\title{
La muralla renacentista de Peñíscola, obra de Vespasiano Gonzaga y Bautista Antonelli $\mathrm{M}^{\mathrm{a}}$ Josefa Balaguer Dezcallar \\ Universitat Politècnica de València, Valencia, España, mabadez@cpa.upv.es
}

\begin{abstract}
Peniscola's fortification is the will of two of the greatest experts in military architecture who worked for Felipe II: Vespasiano Gonzaga, Prince of Sabbioneta, and the engineer Bautista Antonelli. Both of them projected fortifications of great beauty, strength and efficacy and devoted most of their life to the defence of the coastline and borders of the territory of the Spanish monarchy in the second half of the XVI century. The result of this collaboration is described in these pages, together with some drawings of their preserved work and the interpretation of how some elements may have been transformed or even lost according to researchers conducted in recent years.
\end{abstract}

Keywords: fortification, transformations, military architecture

\section{Introducción}

La fortificación de Peñíscola es testimonio del trabajo de dos grandes expertos en arquitectura militar que trabajaron al servicio de Felipe II: Vespasiano Gonzaga y Bautista Antonelli. Ambos dedicaron gran parte de su vida a la defensa de las costas y fronteras de los territorios de la monarquía hispánica en la segunda mitad del siglo XVI y proyectaron fortificaciones de indudable valor artístico y técnico. Para Gonzaga, Peñíscola cierra una etapa de gran actividad en España, durante la cual había sido responsable de las defensas del litoral de Levante, virrey de Navarra y virrey de Valencia En 1578 regresó a sus dominios en Italia y confió al ingeniero Bautista Antonelli el acabado de la obra que se prolongó hasta 1579.Antonelli, estuvo al frente de la obra como director de la ejecución. Antes había acompañado a Gonzaga y a su hermano mayor Juan Bautista Antonelli en sus proyectos en la península y norte de África. En toda esta fase estuvo detrás de ellos aprendiendo a ser ingeniero. En Peñíscola, al frente de la obra, consolidó su experiencia y pudo demostrar su talento y capacitación profesional. Un año después Felipe II le encomendó la realización de planes y obras defensivas en las costas americanas en las que desarrolló criterios y técnicas aprendidas en el Mediterráneo junto a su maestro Gonzaga. Peñíscola supone el fin de esta colaboración y un precedente de la obra posterior de Bautista Antonelli en el Caribe.Ambos, Gonzaga y Antonelli, dejaron su huella en esta pequeña península de Levante creando una obra defensiva de gran belleza que, aunque no se pudo concluir totalmente según el proyecto inicial, protegió a la población en las sucesivas guerras y hoy es símbolo de identidad de la ciudad.

\subsection{Vespasiano Gonzaga (1531 Fondi, 1591 Sabbioneta)}

Pertenecía a la noble familia de los Gonzaga, una de las más influyentes de Italia. Fue príncipe de Sabbioneta, pequeño ducado de Lombardía.

Se formó desde niño en los ambientes más cultos de la Italia del siglo XVI (Nápoles, Roma y 
Mantua) y pasó algunos años en Valladolid en la corte española junto al joven príncipe Felipe.

Aprendió arquitectura, arte, ciencias y técnicas de defensa, que eran materias de estudio para nobles y reyes.

Fué capitán general de la infantería italiana en el Piamonte y Lombardía durante el reinado de Carlos I. En los años sesenta, como Maestre Racional, reconoció el reino de Valencia junto al ingeniero Juan Bautista Antonelli proponiendo proyectos de fortificación en muchos puntos (Boira,1999). También recorrieron el reino de Murcia y planificaron las defensas de Cartagena. En 1571 Gonzaga fue nombrado virrey de Navarra y en 1574 recorrió las fortificaciones de Oran, Mazalquivir, Melilla, Gibraltar y la costa de Andalucía hasta la ciudad de Cádiz. En 1575 fue nombrado virrey de Valencia, cargo que estuvo a punto de rechazar por llevar ya mucho tiempo alejado de sus posesiones italianas, finalmente desempeñó el cargo entre 1575 y 1578.

Del paso de Vespasiano Gonzaga por territorios valencianos, quedó la fortificación de Peñíscola como su obra principal. Además impulsó la reforma del castillo de Alicante revisando las propuestas de otros ingenieros y diseñando algunas de sus trazas. También ideó un castillo para defender la ciudad de Valencia que no se llegó a realizar.

Dejó informes exhaustivos de todo el territorio con vistas de gran interés de ciudades y puertos. En 1575 impulsó la construcción de varias torres nuevas en la costa(Piles y Cullera) y proyectos como el de la torre de Nules que no se realizaron en ese momento. Apoyó inversiones dirigidas a la mejora de la defensa terrestre, frente a inversiones en defensa naval (Belchi, 2006).

Gonzaga fue un gran erudito, amante del arte, de la antigüedad clásica y promotor de la cultura, un humanista del renacimiento que dejó un importante patrimonio cultural. Entre 1556 y 1590 proyectó y construyó la ciudad de Sabbioneta, capital de su pequeño ducado. Fundó esta ciudad fortificada sobre un antiguo castillo situado en una gran llanura, adoptando un trazado de planta hexagonal y baluartes en los ángulos. En esta empresa involucró a ingenieros, artistas y arquitectos tan importantes como Scamozzi. Algunos autores reconocen la influencia de Cattaneo, en cuyo tratado publicado en Venecia en esa época aparecen fortalezas urbanas de trazas similares. Durante largos periodos tuvo que ausentarse de Sabbioneta, pero allí acabó sus días. La ciudad fue considerada por la Unesco modelo de ciudad ideal del renacimiento y declarada Patrimonio de la Humanidad.

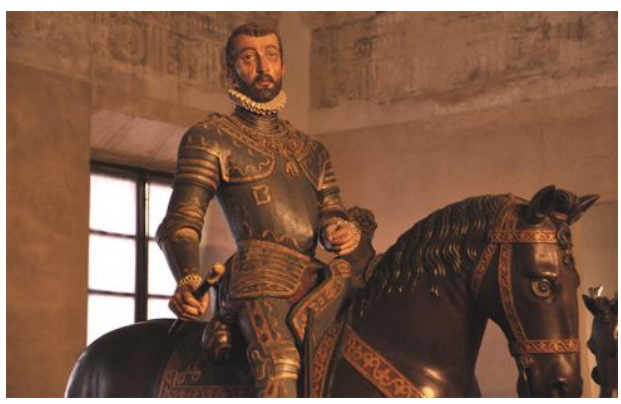

Fig. 1- Estatua ecuestre de V. Gonzaga. Palacio ducal de Sabbioneta ( $\mathrm{M}^{\mathrm{a}}$ Josefa Balaguer, 2014)

Marcó las directrices de las trazas en muchas fortificaciones de la monarquía hispánica en el Mediterráneo. Para ello dirigió a ingenieros sobre aspectos funcionales pero también sobre la materia y la forma. En ocasiones discrepó y les criticó duramente por aplicar soluciones poco adaptadas a la naturaleza, o compitió con ellos ante el rey presentando propuestas alternativas .

En Peñíscola plantea la reforma de las defensas medievales y está seguro de lo que quiere. Se reconoce como responsable y único autor de la traza ante el rey, afirmando en una carta que le envía en abril de 1578, que no ha tenido ningún ingeniero detrás "sy no haviese açertado toda la culpa sera mia porque no e tenido otro ingeniero a la oreja como en otras"1.

Suponemos que para él fue un reto poder aplicar sus ideas sobre la fortificación adaptada al lugar sirviéndose de la naturaleza, de la arquitectura y del arte para crear una imagen imponente visible desde lejos, en la que logra transmitir la idea de protección y del gran poder del imperio. 
Para ello plantea un gran frente a tierra flanqueado por baluartes, semibaluartes $\mathrm{y}$ casamatas protegiendo los flancos, que se van adaptando al peñón. El conjunto forma un gran basamento en la parte inferior del castillo medieval que corta el acceso por tierra y se adelanta respecto a la muralla antigua situando baterías y áreas de maniobra militar en los extremos, separadas del caserío.

En el proyecto integra el mar y las rocas labrando en ellas la base de los baluartes que se adaptan y funden con el peñón en algunas zonas creando un conjunto de gran valor arquitectónico y paisajistico.

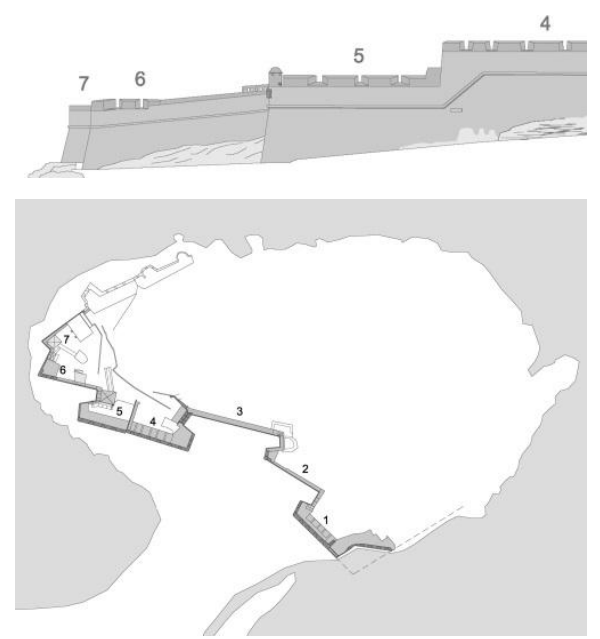

Figs. 2 y 3- Alzado arriba y a la izqda. planta esquemática del frente de tierra. Las denominaciónes han variado,y según la documentación de Simancas es la siguiente: 1.Baluarte de Santa María, 2.Cortina de San Felipe(actualmente Balcón de Pilatos o Mura del Ministre), 3.Cortina firme del frente de tierra, $4 \mathrm{y}$ 5.Baluarte Real (con dos plazas alta y baja que después tomarán el nombre de Batería del Calvario y de Santiago, 6. Punta de San Nicolás, 7. Cortina del Olvido. (M Josefa Balaguer-Vicén, 2013)

Descubrimos en las proporciones y diseño de la portada monumental las directrices dadas por Serlio para las puertas de las ciudades. Para resolver las cartelas y garitas se reinterpretan elementos de la antigüedad clásica y se utilizan formas y técnicas renacentistas (Balaguer, 2013).

Para hacer rústico el almohadillado de la portada se labran bolas, motivo particular de Peñíscola que hemos visto también en el Palacio ducal de Mantua, una obra de Giulio Romano que Gonzaga conocía bien y en la que suponemos pudo inspirarse.
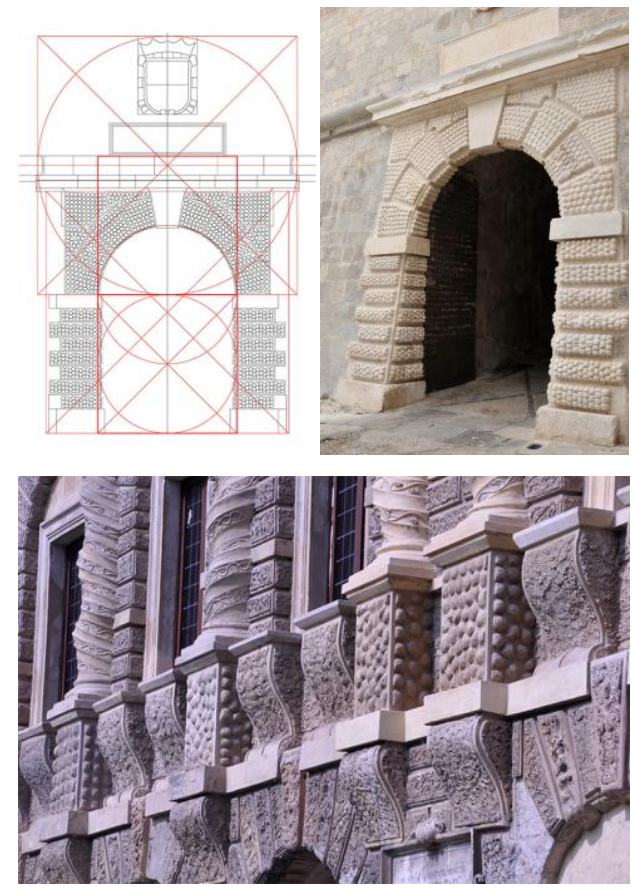

Figs. 4, 5 y 6- Foto y dibujo de la portada de Peñíscola, con estudio de proporciones y abajo detalle del ornamento del Palacio Ducal de Mantua de 2014 (Ma Josefa Balaguer - L. Vicén)

Gonzaga en Peñíscola tuvo un excelente equipo y pudo desarrollar la obra en tan sólo tres años. Bautista Antonelli al frente y detrás de todas las decisiones y como maestro mayor Joan de Ambuesa, cantero de origen francés que había trabajado con ambos en las torres de costa (Arcineaga, 2001) y suponemos que fue fundamental para dirigir los trabajos de cantería que son de gran calidad, después de Peñíscola trabajó en la fase final de la construcción del Monasterio de San Miguel de los Reyes demostrando su gran capacidad técnica. 


\subsection{Bautista Antonelli (Gatteo 1547?-Madrid 1616)}

Bautista Antonelli nació en Gatteo (Italia), llegó a España cuando tenía aproximadamente veinte años. En su trayectoria distinguimos una primera etapa de formación junto con su hermano mayor el ingeniero Juan Bautista Antonelli y Vespasiano Gonzaga en las fortificaciones de la costa peninsular y del norte de África que culmina al abordar la obra de Peñíscola en 1576 para la que fue nombrado director de la obra como ingeniero del rey, firmando todas las certificaciones entre 1576 y 1579. En 1580, ya probada su valía, el rey le encomienda importantes misiones para los planes de defensa de las costas americanas. La primera expedición, en 1581, al estrecho de Magallanes fracasa por un naufragio. Regresa a Madrid donde pasa unos años sin actividad destacada. En 1585 emprende su nueva misión americana y ese mismo año nace su hijo, Juan Bautista, al que después enseñará el oficio de ingeniero.

Será entre 1586 y 1599 la etapa profesional más fecunda de Bautista Antonelli, reconociendo los extensos territorios de la corona en América, dando soluciones y dibujando trazas de muchas fortificaciones, que a partir de los años 90 ya firmará como autor: La Habana, Santiago de Cuba, Puerto Rico, Panamá, Veracruz, Santo Domingo, etc., obras de un valor excepcional.

Entre 1599 y 1603 regresa a Madrid y trabaja unos años en las costas de Levante, Gibraltar y África. Vuelve a América en 1603 y en 1604 deja a su hijo en la Habana como encargado de continuar sus trabajos.

Diego Angulo, su biógrafo destacó su labor excepcional en esta etapa que lo convierte en el "gran ingeniero del siglo XVI en indias"(Angulo 1942). En los últimos 12 años de su vida ya no vuelve a América, pero sigue trabajando en las fortificaciones de Gibraltar y Larache durante cinco años, participando además en la toma de esta ciudad al entrar disfrazado como espía para tomar datos sobre sus defensas.

Muere solo en Madrid en 1616, tras una vida muy dura al servicio de la corona, recorriendo gran parte del continente americano, España y el norte de África, con misiones que lo mantuvieron alejado de su familia y en ocasiones gravemente enfermo. Dejó un patrimonio fortificado de gran valor cultural testimonio de su talent.

\section{Interpretación de los planos del proyecto}

En el Archivo de Simancas se encuentran los dos planos del proyecto de Gonzaga, datados en 1579 , en la fase final de la construcción de la muralla, conteniendo ambos una alternativa a la finalización de la muralla en el frente sur, que no se acabó según la propuesta de Gonzaga. Se trata de las más antiguas representaciones técnicas de la fortaleza que se han encontrado y constituyen un documento de gran valor para la investigación de la fortificación de Peñíscola.

Estos planos no están firmados ni fechados pero los hemos contrastado con otros firmados por Bautista Antonelli años después en el Nuevo Continente (proyecto de Santo Domingo, etc.), coincidiendo la caligrafía y el estilo del dibujo.

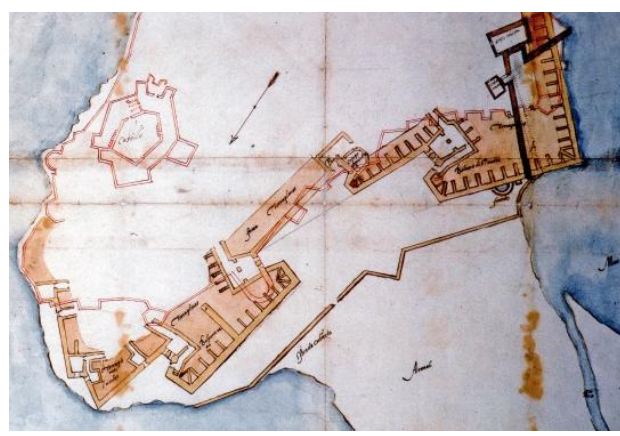

Fig. 7- Archivo General de Simancas (AGS) MPD, 09,059 (Plano de Peñíscola)

La perfección del plano refleja un conocimiento profundo del proyecto, la realidad y la obra realizada, que es difícil imaginar en otro distinto del que fue en todo momento el máximo responsable de las obras. Aparte de la autoría intelectual del proyecto, que se debe atribuir a Vespasiano Gonzaga, se puede asegurar que Bautista Antonelli además de dirigir la obra y dar solución a su terminación fue el autor de estos planos.

La mayor parte del conocimiento que hoy tenemos de la fortificación medieval y de la 
muralla moderna lo hemos podido obtener a partir de la interpretación de estos planos, que no dejan de sorprendernos por la exactitud de su realización y la precisión de su descripción gráfica.

La diferenciación por colores de la muralla vieja y la muralla nueva nos permite reconocer el aspecto que tendría el recinto medieval e identificar algunas obras de fortificación realizadas durante el reinado de Carlos I.

La elección del plano de corte de la planta nos da una exhaustiva información sobre el sistema constructivo, basado en estribos transversales en las partes mas altas y terraplenos. También nos permite conocer el sistema de acceso de la artillería a las casamatas en los flancos de los semibaluartes y los ángulos de las tijeras.

Los dos planos contienen prácticamente la misma descripción de la muralla, pero el segundo está más centrado en el debate que se estableció sobre el acabado de la obra entre el ingeniero Fratín y Bautista Antonelli, otro argumento más que confirmaría su autoría ya que los textos incluidos en el plano reproducen las mismas ideas que firma Antonelli en los legajos que acompañan los planos.
En el segundo plano se representa la propuesta que hizo el ingeniero Fratín y se ve la problemática que suponía realizar la solución alternativa que propuso para poder acabar la muralla por el Sur sin tener que entrar en el mar, pues dejaba fuera el manantial que abastecía a la población y se demolía un tramo de la muralla medieval y un conjunto de casas. Propuesta que gracias a los argumentos que expuso Antonelli en los informes que se conservan en Simancas fue reconsiderada y no se ejecutó (Balaguer, 2012).

En estos últimos años hemos digitalizado los planos de Simancas y cruzado con los informes que los acompañan. De este estudio ha salido la denominación original de los diversos elementos que componen la intervención y la localización de elementos de interés que se han podido recuperar o investigar en la restauración.

\section{Investigaciones sobre los remates de la muralla}

La obra no se concluyó según el proyecto original quedando inacabados el baluarte de Santa María en el extremo sur y también algunos remates.

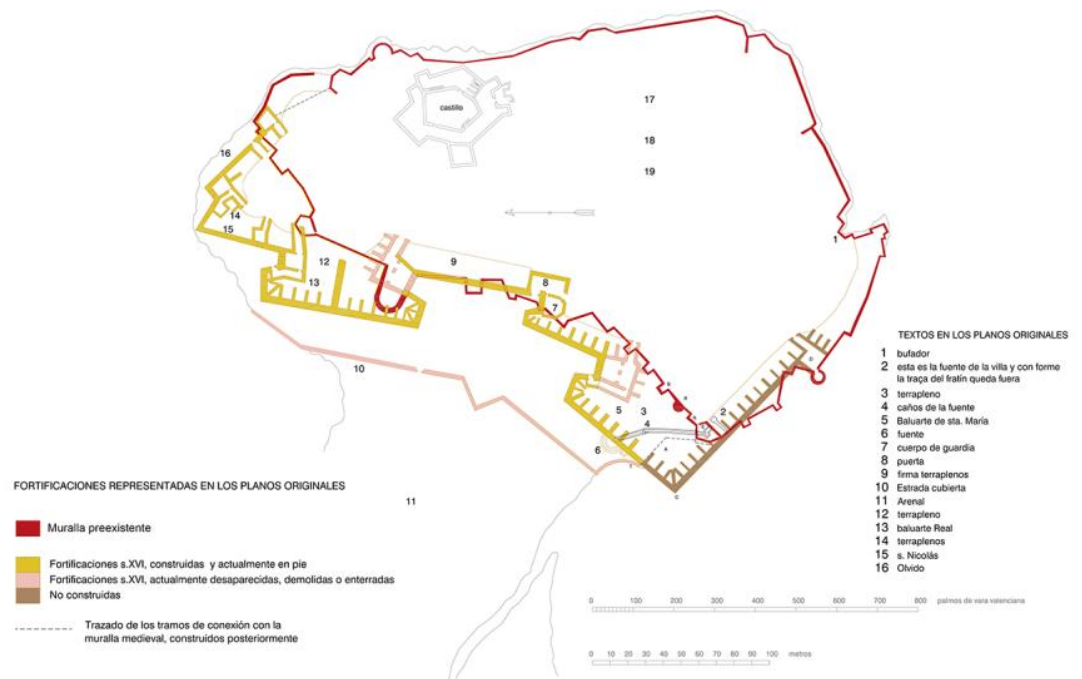

Fig. 8- Transcripción e interpretación del plano de Simancas. MPD, 09,059. (Balaguer-Vicén)

En los últimos años hemos investigado sobre cómo pudieron ser estos elementos que no se concluyeron o fueron objeto de remodelaciones posteriores (Balaguer-Vicén, 2013). Algunos resultados y su representación gráfica se describen a continuación. 


\subsection{Las garitas}

Solo se conserva una garita de la obra original, la llamada garita de Felipe II, a la derecha del acceso a la fortaleza, en la esquina sobre el escudo. Tiene cúpula semiesférica de cantería y es un modelo de estereotomía renacentista por su diseño y el perfecto encaje de las grandes piezas que forman esta pequeña construcción. Hubo otra garita igual en la esquina norte que fue abatida en la Guerra de la Independencia, posteriormente reconstruida en el siglo $\mathrm{XX}$ conserva vestigios de la original que hemos dejado a la vista al restaurar esa zona en 2013.

Suponemos que debió haber otra garita en la esquina sur del Baluarte Real, sobre el escudo, ya que aparece dibujada en algunos planos del siglo XVIII (Desnaux, 1747) y probablemente otra sobre el escudo del Baluarte de Santa María. Adjuntamos una hipótesis gráfica del Baluarte Real tal como pudo ser (Fig.16).

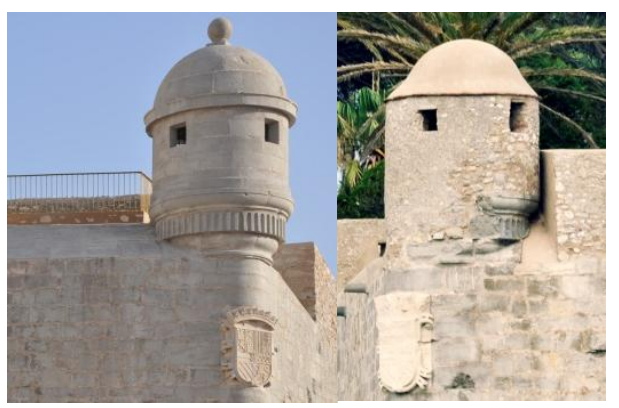

Figs. 8 y 9- A la izquierda garita original y a la derecha otra reconstruida en el siglo XX que conserva parte de la base ( $\mathrm{M}^{\mathrm{a}}$ Josefa Balaguer, 2013)

\subsection{Los parapetos}

Los remates de la muralla no aparecen representados en los planos del proyecto pues son un corte en planta a nivel inferior.

Los remates que se conservan son de mampostería y corresponden a fases posteriores (siglos XVIII-XIX). En las recientes obras de restauración hemos localizado algunos remates de cantería ocultos bajo obras posteriores que fueron remodelados y suponemos que Gonzaga pudo plantear para toda la muralla.
A ambos lados de la garita de Felipe II hay un parapeto corrido abocelado para "tirar a barbeta" formado por grandes piezas de cantería perfectamente engarzadas que se hallaba bajo muretes y rellenos del siglo XIX y que hemos dejado al descubierto.

En el Baluarte Real también se ha localizado, en la plaza superior (Batería del Calvario) parte del parapeto con troneras que quedó fosilizado en otro posterior y que se ha dejado a la vista en la cara interna de la batería (fig.10). No ha sido posible precisar las características de la cara externa (ángulos de tiro y geometría del alambor) pero hemos dibujado una hipótesis sobre la posible configuración inicial del baluarte con los datos recabados. Podemos concluir que los remates de la muralla se concibieron abocelados, con troneras o corridos, similares a los que después realizó Bautista Antonelli en algunas obras del Caribe (El Morro o San Salvador de la Punta en la Habana).

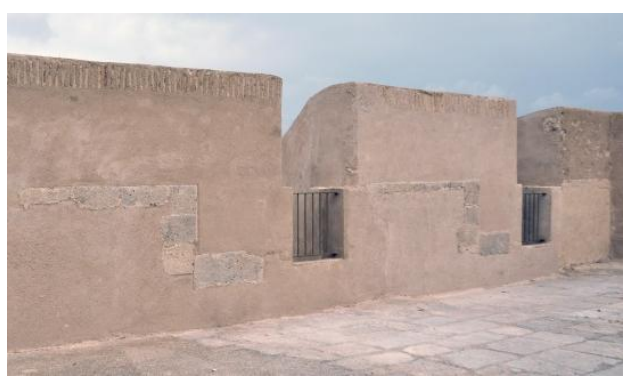

Fig. 10- Foto de parapeto del Baluarte Real con restos del parapeto original localizados bajo los revestimientos modernos $\left(\mathrm{M}^{\mathrm{a}}\right.$ Josefa Balaguer, 2013)

\subsection{Las casamatas de la muralla}

En el proyecto se plantearon dos tipos de casamatas. Unas más bajas que actualmente se conservan en la zona norte, en la punta de San Nicolás y en la cortina del Olvido a las que se accede por túneles y rampas, con troneras semicubiertas similares a las proyectada por Escrivá en San Telmo en Nápoles (Cobos y Castro, 2000).

Otro tipo fueron las realizadas para defender los flancos en los baluartes de Santa María y Real actualmente muy transformadas. Éstas se 
plantearon con dos niveles y dos troneras separadas por un merlón central con derrame curvo según hemos podido constatar al estudiar el Baluarte Real. La casamata recayente al sur de este baluarte fue demolida parcialmente a principios del siglo XX para hacer una escalera de acceso a unas escuelas pero se conserva una fotografía anterior (Fig.13).

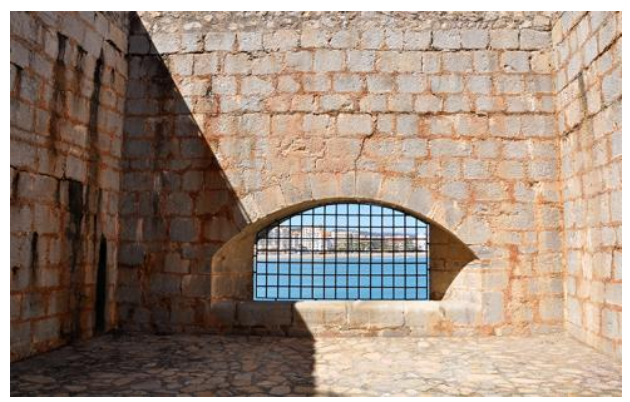

Fig. 11- Tronera en la cortina del Olvido

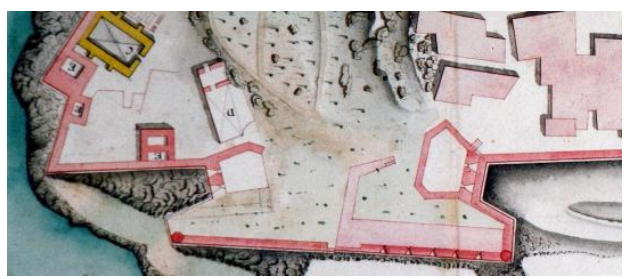

Fig. 12- Fragmento de plano del "Proyecto de un Almacén de Pólvora a prueba de bomba". 1.747 Carlos Desnaux. AGS. MPD, XVIII, 259

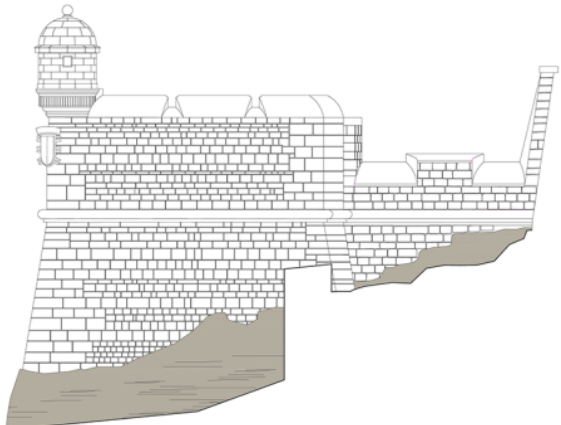

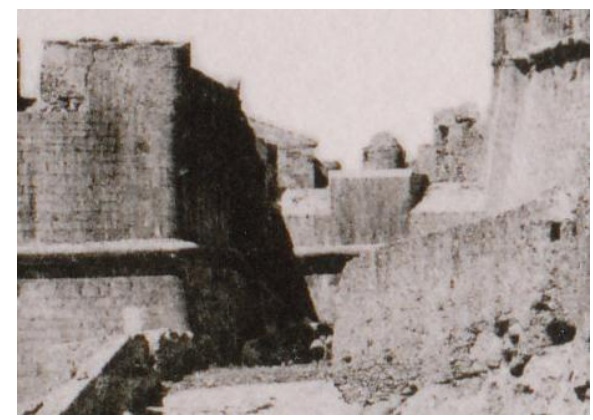

Fig. 13- Izda. Fotografia de principios de siglo XX donde aparece la casamata del baluarte real recayente a la rampa antes de demolerse

Detrás del parapeto hemos localizado enterrados los muros de cierre de esta casamata que finalmente no se llegaron a levantar (F.Blay/MJ. Balaguer). En base a los vestigios, niveles y fotos aportamos una hipótesis de reconstrucción de la casamata y remates de esta zona (Fig. 15)

Forma en planta un hexágono que no se corresponde exactamente con el dibujo del plano de Simancas pero encaja mejor con la representación que aparece en los planos del siglo XVIII (C. Desnaux)

\section{Notas}

(1) Archivo de Simancas, IX-57; G.A., leg 90, 20 a 29. Escrito de Vespassiano Gonçaga a su majestad (S.C.R.M.) de 15 de abril 1578 .

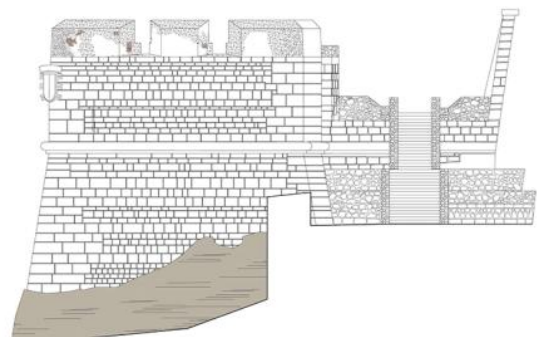

Fig. 14 y 15. Dibujos comparativos de la casamata del Baluarte Real. A la izquierda estado actual y a la derecha hipótesis de reconstrucción gráfica en base a los vestigios localizados, representando la garita que pudo haber (Balaguer) 


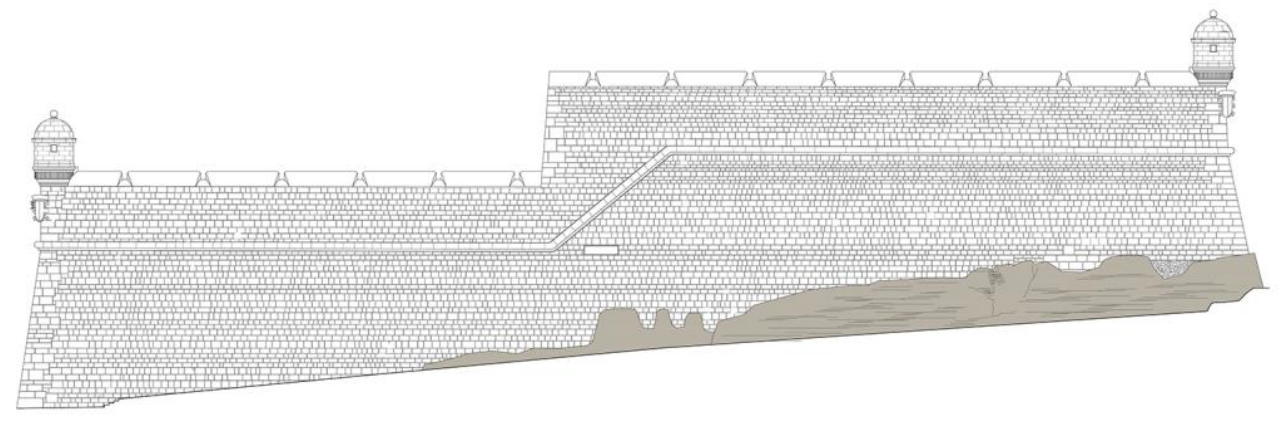

Fig. 16- Frente del Baluarte con hipótesis sobre cómo pudieron concebirse los remates en el proyecto de Gonzaga, en base a la tipología localizada en el extremo sur (Balaguer 2015)

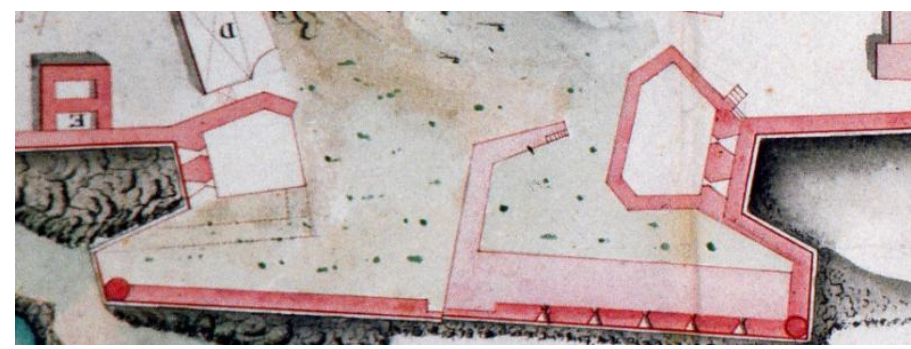

Fig. 17- Detalle del plano de Carlos Desnaux de 1747 ya citado, donde representa el Baluarte Real con las dos garitas y las casamatas en el siglo XVIII, con los parapetos posiblemente ya reformados, dado que en el lado derecho hemos localizado otro distinto con troneras que es el que se ha representado en la Figura 15

\section{Referencias}

Arcineaga, L. (2001) El Monasterio de san Miguel de los Reyes. Biblioteca Valenciana. Ed. pp. 230236.

Angulo, D., Sánchez, F.J. (1942). Bautista Antonelli. Las fortificaciones americanas del sigloXVI Discurso de ingreso de Diego Angulo Iñiguez y contestación de F.J. Sánchez Cantón. Real Academia de la Historia. Ed. Madrid.

Balaguer, $\mathrm{M}^{\mathrm{a}} \mathrm{J}$. (2012). La restauración de las fortificaciones de Felipe II en Peñíscola. En Actas del IV congreso de Castellología, edición digital. Asociación Española de Amigos de los castillos. AEAC. Ed. Madrid. pp. 1173-1191.

Balaguer, $\mathrm{M}^{\mathrm{a}} \mathrm{J}$. (2013) Ingenio y Arte en la fortificación de Peñíscola en Las fortificaciones de los Antonelli en Cuba. Siglos XVI-XVII.Germán García Segura. Ed. España. pp. 61-65.

Balaguer, M ${ }^{a}$ J., Vicén, L. (2013). La muralla renacentista de Peñíscola. Caracterización de elementos materiales y sistemas constructivos. En Actas del VIII Congreso de Historia de la Construcción Volumen I. Santiago Huerta, Fabian López Ulloa. Ed. Madrid, pp. 73-82.

Belchi, M. (2006). Felipe II y el virreinato valenciano (1567-1578): la apuesta por la eficacia gubernativa. Biblioteca Valenciana Ed. Valencia.

Boira Maiques, J. (1999). "Geografía y control del territori. El coneiximent $i$ la defensa del litoral Valencià al segle XVI: L'enginer Joan Baptista Antonelli” en Cuadernos de Geografía $n^{\circ}$ 52. Ed. Valencia. pp. 183-189.

Cobos F., Castro J. (2000). Luis Escrivá. Su apología y la fortificación imperial. Biblioteca Valenciana. Ed., Valencia. 